\title{
Accuracy Enhancement of Parameter Estimation and Sensorless Algorithms Based on Current Shaping
}

\author{
Jin-Woong Kim ${ }^{*}$ and Jung-Ik $\mathrm{Ha}^{\dagger}$ \\ ${ }^{*}$, School of Electrical and Computer Engineering, Seoul University, Seoul, Korea
}

\begin{abstract}
Dead time is typically incorporated in voltage source inverter systems to prevent short circuit cases. However, dead time causes an error between the output voltage and reference voltage. Hence, voltage equation-based algorithms, such as motor parameter estimation and back electromotive force (EMF)-based sensorless algorithms, are prone to estimation errors. Several dead-time compensation methods have been developed to reduce output voltage errors. However, voltage errors are still common in zero current crossing areas, and an effect of the error is much worse in a low speed region. Therefore, employing voltage equation-based algorithms in low speed regions is difficult. This study analyzes the conventional dead-time compensation method and output voltage errors in low speed operation areas. A current shaping method that can reduce output voltage errors is also proposed. Experimental results prove that the proposed method reduces voltage errors and improves the accuracy of the parameter estimation method and the performance of the back EMF-based sensorless algorithm.
\end{abstract}

Key words: Back EMF-based sensorless, Current shaping, Online parameter estimation, Output voltage error, Permanent magnet synchronous motor

\section{INTRODUCTION}

Permanent magnet synchronous machines (PMSMs) are used in many applications because of their high efficiency, power density, and torque to current ratio. The high performance control of PMSMs requires rotor angle information and machine parameters. Rotor angle information can be easily obtained from encoders or resolvers. However, encoders are not allowed in some applications because of cost or space issues [1]. Thus, sensorless algorithms, such as the back electromotive force (EMF)- or saliency-based methods, are required in rotor angle estimation [2], [3]. Machine parameters, such as rotor flux linkage, stator resistance, and inductance are considered as constant values. However, the values of such parameters vary with operation conditions and aging processes [4]. For example, stator winding resistance and rotor flux linkage are affected by temperature [5]. Stator resistance tends to increase as winding temperature increases. Rotor flux linkage tends to decrease as the temperature of a permanent magnet

Manuscript received Oct. 26, 2015; accepted Nov. 18, 2015

Recommended for publication by Associate Editor Kwang-Woon Lee.

${ }^{\dagger}$ Corresponding Author: jungikha@snu.ac.kr

Tel: +82-880-1760, Fax: +82-878-1452, Seoul Nat'l University

*Dept. of Electrical and Computer Eng., Seoul Nat'l University, Korea increases. The inductances of machines change according to magnetic saturation [6]. Any variation in parameters can seriously affect control performance and efficiency. Hence, parameters need to be estimated in real time and updated on the controller for performance improvement.

Back EMF-based sensorless algorithms and online parameter estimation algorithms are based on the voltage equation of electric machines [7]-[9]:

$$
\begin{aligned}
& v_{d s}^{r}=R_{s} i_{d s}^{r}+L_{d} \frac{d i_{d s}^{r}}{d t}-\omega_{r} L_{q} i_{q s}^{r} \\
& v_{q s}^{r}=R_{s} i_{q s}^{r}+L_{q} \frac{d i_{q s}^{r}}{d t}+\omega_{r}\left(L_{d} i_{d s}^{r}+\lambda_{p m}\right)
\end{aligned}
$$

where $R_{s}$ is the stator resistance, $L_{d}$ and $L_{q}$ are the inductances, $\lambda_{p m}$ is the flux linkage constant, and $\omega_{r}$ is the electrical speed of the motor. In (1), information on the output voltage and output current is required for the parameter estimation method or sensorless algorithm. Current information can be obtained using a current sensor or shunt resistor. However, obtaining output voltage information in a general voltage source inverter (VSI) system is considerably difficult. Hence, the output voltage reference is used instead of the output voltage. However, voltage errors may lead to parameter estimation errors when the output voltage and voltage reference are different as a result of the nonlinearity of VSI 
systems. The effect of such nonlinearity on low speed regions is relatively more severe than that on mid and high speed regions because of the small values of voltage references [10]. Thus, employing a sensorless algorithm or parameter estimation algorithm in a low speed region is challenging. The nonlinearity of VSI systems can be reduced using the dead-time compensation algorithm if the current magnitude is sufficiently large. In this way, rotor angles or parameter estimation errors can be reduced. However, compensation may not be achieved at zero current crossing areas because of parasitic capacitance and current direction errors.

A method for reducing voltage errors using a current shaping technique is proposed in the present study. Specifically, this study focuses on error-causing areas near zero current points [11], [12]. It also analyzes the conventional dead-time compensation method and the voltage errors in operation areas. The proposed method can reduce voltage errors by causing each phase current to change rapidly as currents cross the zero point. Current shaping is implemented via d-axis current injection. Hence, the adjustment of each phase current reference has no effect on the q-axis current reference, and no additional torque ripple occurs. The proposed method can be easily incorporated into the conventional dead-time compensation method. The feasibility of the proposed method and the accuracy enhancement of the parameter estimation method and sensorless algorithm in low speed regions are shown through experimental results using a surface mounted permanent magnet (SMPM) machine.

This paper is organized as follows. The analysis of output voltage errors is introduced in Section II. The proposed current shaping method for output voltage error reduction is presented in Section III. The experimental results of the parameter estimation and sensorless algorithms are illustrated in Section IV. Some conclusions are given in Section V.

\section{ANALYSIS OF VOLTAGE ERRORS}

\section{A. General Dead-time Compensation Method}

The conventional dead-time compensation method generally involves adjusting gating signals to make the output voltage and voltage reference equal during one sampling period [13]. Fig. 1 illustrates an a-phase current flow and adjusted gating signals when the current $i_{a}$ flows to the machine. As shown in Fig. 1(a), the current flows through $\mathrm{D}_{\mathrm{a}}$ during dead time. The output voltage is $\mathrm{V}_{\mathrm{dc}}$ when $\mathrm{S}_{\mathrm{a}}$ is in the on state, whereas it is equal to zero when $\mathrm{S}_{\mathrm{a}}$ is in the off state. The output voltage is determined regardless of the state of $S_{a}$. As shown in Fig. 1(b), the gating signal is adjusted to make the output voltage and voltage reference equal. Compensation is not required for the zero output voltage. By contrast, $S_{a}$ needs to be turned off earlier than the ideal gating signal for the $\mathrm{V}_{\mathrm{dc}}$ output voltage.

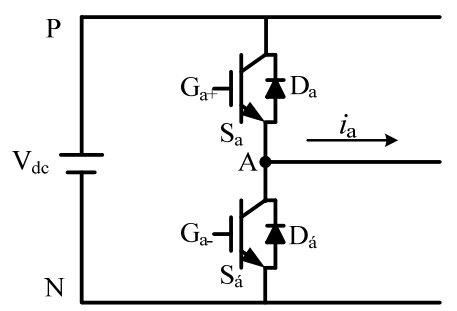

(a)

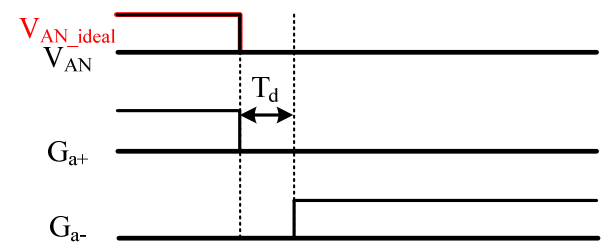

(b)

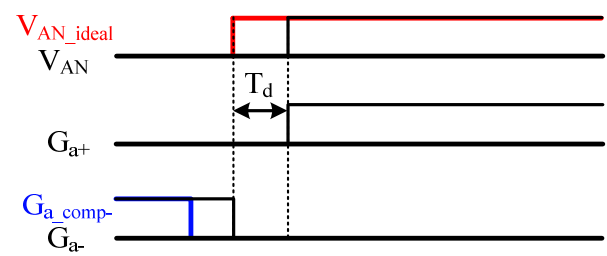

(c)

Fig. 1. Dead-time compensation at a positive current. (a) Current flow of a phase. (b) Compensation in the off state. (c) Compensation in the on state.

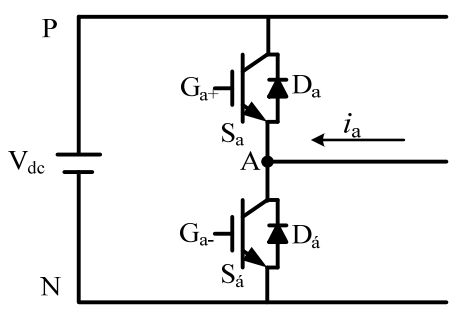

(a)

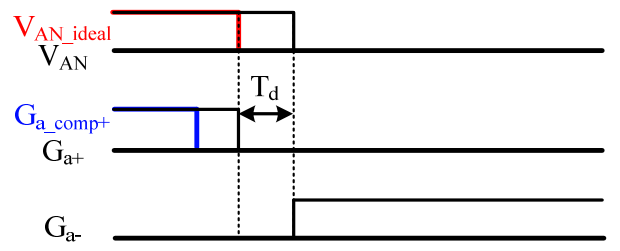

(b)

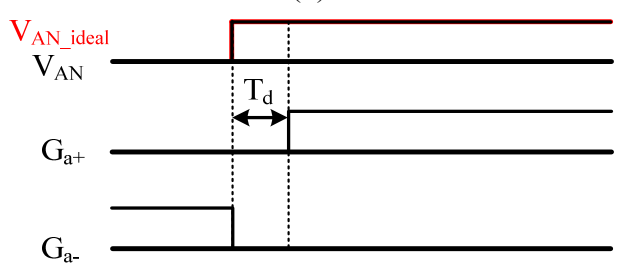

(c)

Fig. 2. Dead-time compensation at a negative current. (a) Current flow of a phase. (b) Compensation in the off state. (c) Compensation in the on state.

Fig. 2 shows an a-phase current flow and adjusted gating signals when the current $i_{a}$ flows from the machine. The current flows through $\mathrm{D}_{\mathrm{a}}$ during dead time. The output 


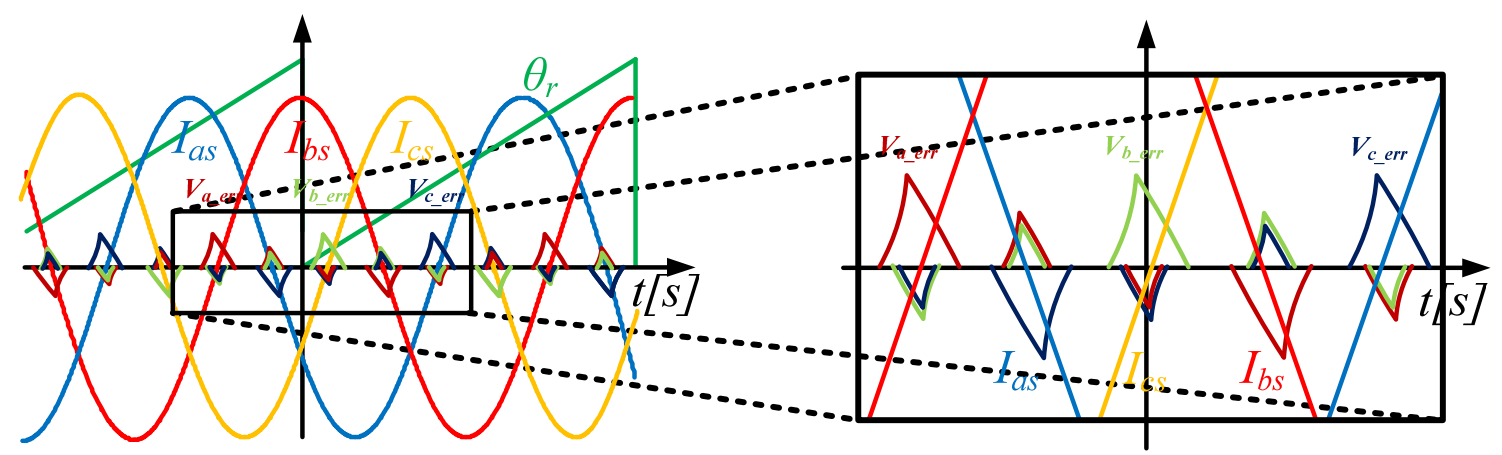

(a)

(b)

Fig. 3. Compensation with the conventional method. (a) Current waveform. (b) Enlarged view of a voltage error.

voltage is zero when $\mathrm{S}_{\mathrm{a}}$ is in the on state, whereas it is $\mathrm{V}_{\mathrm{dc}}$ when $S_{a}$ is in the off state. The output voltage is determined regardless of the state of $S_{a}$. As shown in Fig. 2(b), the gating signal is adjusted to compensate for the voltage error. $\mathrm{S}_{\mathrm{a}}$ needs to be turned off earlier than the ideal gating signal for the zero output voltage. By contrast, compensation is not required for the $\mathrm{V}_{\mathrm{dc}}$ output voltage. Even with the application of the above compensation method, output voltage errors can still be found in zero current crossing areas because of parasitic capacitance and current direction errors.

\section{B. Output Voltage Error Analysis}

The proposed method focuses on error-causing areas near zero current points to reduce output voltage errors. Sinusoidal currents slowly change when they pass the zero current point, as shown in Fig. 3(a). Hence, voltage errors cannot be eliminated even with the application of the dead-time compensation method. The estimated output voltage and the error between the reference voltage and the estimated one can be calculated with the following voltage equation if the motor parameters and rotor position are known:

$$
\begin{gathered}
\hat{v}_{d s}^{r}=R_{s} i_{d s}^{r}+L_{d} \frac{d i_{d s}^{r}}{d t}-\omega_{r} L_{q} i_{q s}^{r} \\
\hat{v}_{q s}^{r}=R_{s} i_{q s}^{r}+L_{q} \frac{d i_{q s}^{r}}{d t}+\omega_{r}\left(L_{d} i_{d s}^{r}+\lambda_{p m}\right) \\
v_{d s_{-} e r r}^{r}=v_{d s}^{r^{*}}-\hat{v}_{d s}^{r} \\
v_{q s_{-} e r r}^{r}=v_{q s}^{r^{*}}-\hat{v}_{q s}^{r}
\end{gathered}
$$

where ${ }^{\wedge}$ denotes the estimated value. The other symbols have been defined previously. As shown in Fig. 3(b), the errors of each output voltage become large when each phase current passes the zero current point. Hence, this region needs to be reduced or eliminated to achieve an accurate output voltage.

\section{PROPOSED METHOD}

\section{A. Current Shaping Method}

The proposed method changes the waveform of a phase current to reduce the error-causing region. As shown in Fig.

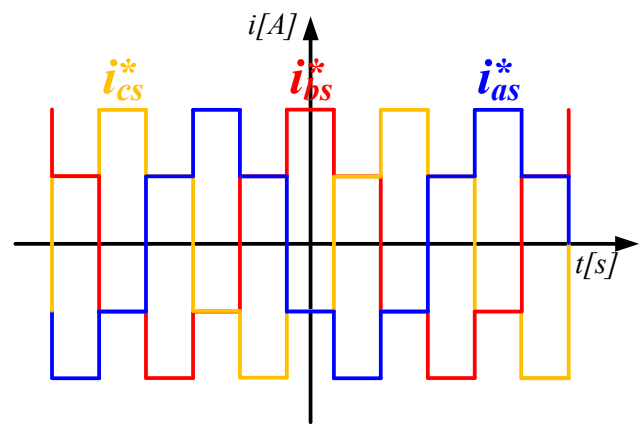

(a)

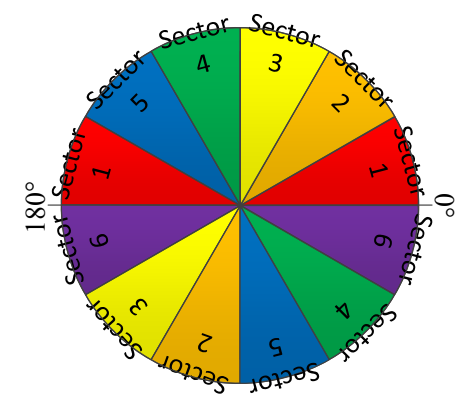

(b)

Fig. 4. Proposed current shaping. (a) Phase current reference. (b) Divided sectors for the proposed method.

4(a), each phase current decreases or increases dramatically when it passes the zero current region. Output voltage errors can be reduced by the proposed current waveform. Without any assumption, many solutions are available to make this type of current waveform. However, the following equations should be satisfied for the preserved q-axis current reference, which is directly related to the output torque:

$$
\begin{aligned}
& I_{q s}^{e^{*}}=-i_{d s}^{s} \sin \theta_{r}+i_{q s}^{s} \cos \theta_{r} \\
& i_{d s}^{s}=\frac{2}{3} i_{a s}^{*}-\frac{1}{3}\left(i_{b s}^{*}+i_{c s}^{*}\right), \quad i_{q s}^{s}=\frac{1}{\sqrt{3}}\left(i_{b s}^{*}-i_{c s}^{*}\right) \\
& i_{a s}^{*}+i_{b s}^{*}+i_{c s}^{*}=0
\end{aligned}
$$

where $I_{q s}{ }^{e^{*}}$ is the determined value from the speed controller or torque controller and the symbol * of each phase current denotes a reference. $\theta_{r}$ represents the electric angle, which is 
TABLE I

References of EACH Phase CURRENT ACCORding to SECtor

\begin{tabular}{|c|c|c|c|c|}
\hline Sector & Electric angle & $i_{a s}^{*}$ & $i_{b s}^{*}$ & $i_{c s}^{*}$ \\
\hline 1 & $\begin{array}{c}0^{\circ}<\theta<30^{\circ} \\
150^{\circ}<\theta<180^{\circ}\end{array}$ & $-k I_{q s}^{e^{*}}$ & $I_{q s}^{e^{*}} \frac{\sqrt{3} k}{2 \cos \theta}\left(\frac{1}{k}-\sin \theta+\frac{\cos \theta}{\sqrt{3}}\right)$ & $-i_{b s}^{*}-i_{c s}^{*}$ \\
\hline 2 & 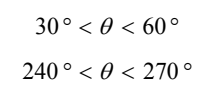 & $I_{q s}^{e^{*}} \frac{\sqrt{3}-2 k \cos \theta}{\sqrt{3} \sin \theta+\cos \theta}$ & $-i_{a s}^{*}-i_{c s}^{*}$ & $-k I_{q s}^{e^{*}}$ \\
\hline 3 & $\begin{aligned} 60^{\circ} & <\theta<90^{\circ} \\
210^{\circ} & <\theta<240^{\circ}\end{aligned}$ & $I_{q s}^{e^{*}} \frac{\sqrt{3}+2 k \cos \theta}{\sqrt{3} \sin \theta+\cos \theta}$ & $-i_{a s}^{*}-i_{c s}^{*}$ & $k I_{q s}^{e^{*}}$ \\
\hline 4 & $\begin{array}{c}90^{\circ}<\theta<120^{\circ} \\
300^{\circ}<\theta<360^{\circ}\end{array}$ & $-I_{q s}^{e^{*}} \frac{\sqrt{3}-2 k \cos \theta}{\sqrt{3} \sin \theta-\cos \theta}$ & $k I_{q s}^{e^{*}}$ & $-i_{b s}^{*}-i_{c s}^{*}$ \\
\hline 5 & 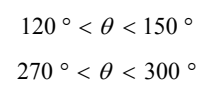 & $-I_{q s}^{e^{*}} \frac{\sqrt{3}+2 k \cos \theta}{\sqrt{3} \sin \theta-\cos \theta}$ & $-k I_{q s}^{e^{*}}$ & $-i_{b s}^{*}-i_{c s}^{*}$ \\
\hline 6 & $\begin{aligned} 180^{\circ} & <\theta<210^{\circ} \\
330^{\circ} & <\theta<360^{\circ}\end{aligned}$ & $k I_{q s}^{e^{*}}$ & $I_{q s}^{e^{*}} \frac{\sqrt{3} k}{2 \cos \theta}\left(\frac{1}{k}+\sin \theta-\frac{\cos \theta}{\sqrt{3}}\right)$ & $-i_{b s}^{*}-i_{c s}^{*}$ \\
\hline
\end{tabular}

obtained from the position sensor. The three unknown variables are $i_{a s}{ }^{*}, i_{b s}{ }^{*}$, and $i_{c s}{ }^{*}$; the number of the given equations is two. Hence, one more assumption or equation is required for the finite solution. As shown in the following equation, one of the phase current references is fixed according to the electric angle:

$$
i_{a s}^{*} \text { or } i_{b s}^{*} \text { or } i_{c s}^{*}= \pm k I_{q s}^{e^{*}}
$$

where $k$ denotes the proportional constant, which varies from 0 to 1 . Other references can be calculated from Equation (6) if one of the current references is fixed. Table I describes the references of each phase current in terms of sector, which is presented in Fig. 4(b).

An additional d-axis current reference is required for the proposed current shaping given that the assumption is the preservation of the q-axis current reference. The shape and magnitude of the d-axis injection and each phase current change according to the proportional constant $k$. Fig. 5 presents the current waveforms of the proposed method according to the $k$ value. On the one hand, the current does not change dramatically near the zero current area if the value of $k$ is too small, as shown in Fig. 5(a). In this case, current shaping becomes useless in the reduction of output voltage errors. On the other hand, the current waveforms become distorted seriously if the value of $k$ is too large, as shown in Fig. 5(c). In this case, the voltage error reduction and the degree of current distortion should be considered in determining the $k$ value. Considering the simplicity of the current shaping realization and injection magnitude, the value of $k$ is determined to be 0.5 in this study.

The proposed references of each phase current can be simplified as d-axis current references with the following equation:

$$
I_{d s}^{e^{*}}=-\frac{I_{q s}^{e^{*}}}{2}+\frac{I_{q s}^{e^{*}}}{60^{\circ}}\left(\theta_{r}-60^{\circ} \times(n-1)\right)
$$

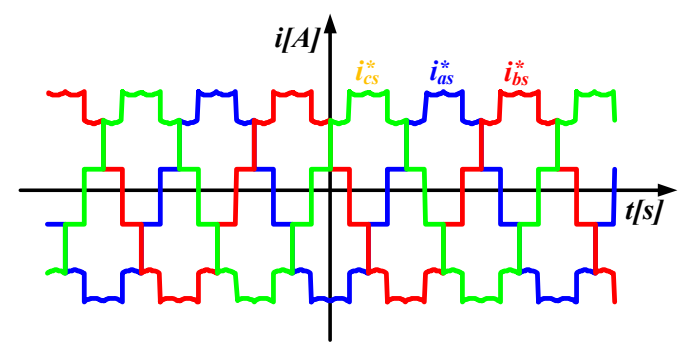

(a)

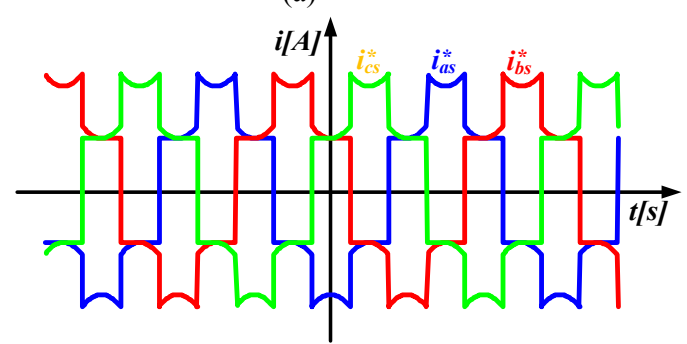

(b)

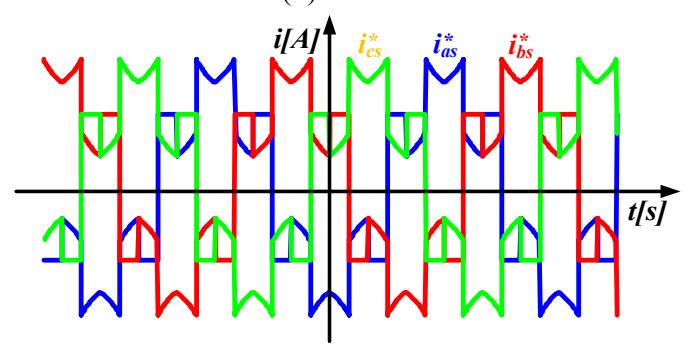

(c)

Fig. 5. Current waveform of the proposed method according to the $k$ value. (a) $k=0.25$. (b) $k=0.5$. (c) $k=0.75$.

where $n$ denotes the sector divided by the electric angle. Such sector is different from the one shown in Fig. 4(b). The sector is redefined in Fig. 6(b) to simplify the d-axis current reference. Fig. 6(a) shows that the proposed d-axis reference represents a sawtooth waveform, the frequency is six times that of the fundamental frequency. Hence, the proposed 


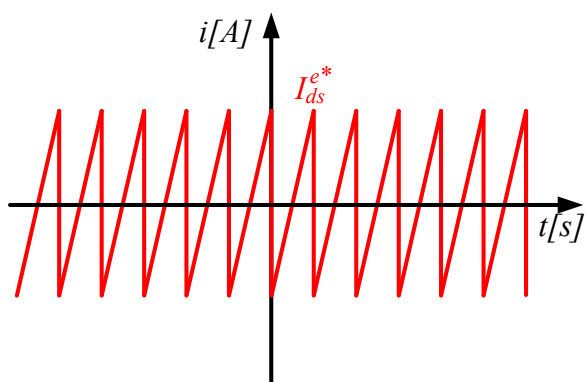

(a)

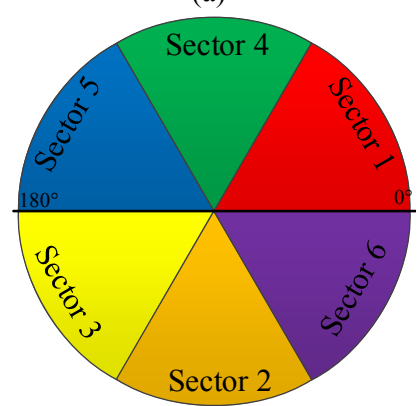

(b)

Fig. 6. Proposed d-axis current injection. (a) D-axis current reference. (b) Divided sectors for the d-axis injection.

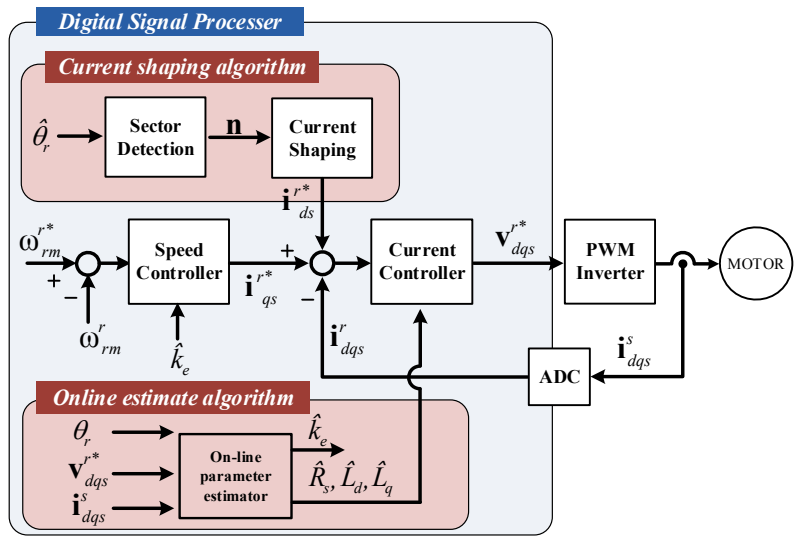

Fig. 7. Proposed control block diagram for the parameter estimation.

current shaping algorithm can be easily implemented using the sawtooth waveform of the d-axis injection. The sector number $n$ is determined from the electric angle, which can be obtained from the encoder or resolver. The sector number is used to establish the d-axis current reference. Such reference is added to the current reference that serves as the output of the speed controller.

\section{B. Parameter Estimation}

The overall control block diagram for the parameter estimation is shown in Fig. 7. The only difference is added block compared with the conventional one. Operation modes are divided according to the motor speed, and the different parameter estimation algorithm is used in each mode. The high frequency signal of the sine or square waveform injection is used for the online parameter estimation in the mid and high speed regions, in which the proposed method is not required.

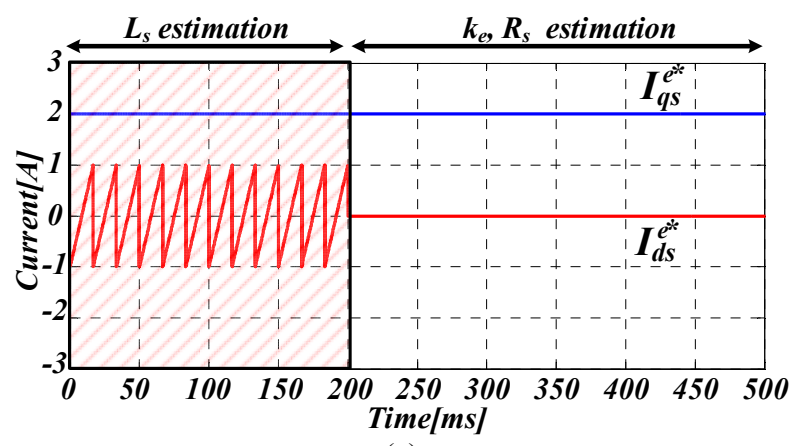

(a)

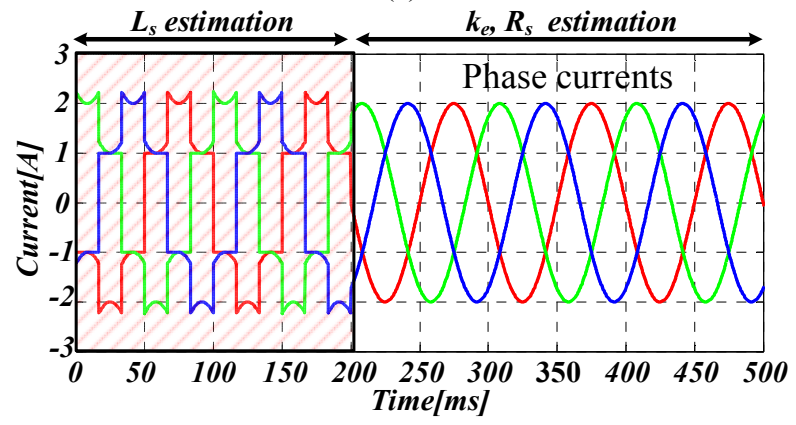

(b)

Fig. 8. Proposed current shaping. (a) Waveform of d, q-axis current. (b) Waveform of phase currents.

As described previously, the proposed current shaping method is used in the low speed region. As shown in Fig. 8, the proposed d-axis current is injected for a period of time when the inductance estimation is required. The output voltage error is then reduced during the injection, thereby enhancing the accuracy of the online parameter estimation. Parameters are estimated on the basis of the recursive least squares (RLS) method, which can be expressed as follows [14], [15]:

$$
\begin{aligned}
Y(k)= & \boldsymbol{\Theta}(k) \mathbf{Z}(k) \\
\hat{\Theta}(k)= & \hat{\Theta}(k-1)+ \\
& \frac{\mathbf{P}(k-1) \mathbf{Z}(k)}{\lambda+\mathbf{Z}(k) \mathbf{P}(k-1) \mathbf{Z}(k)} \times(Y(k)-\mathbf{Z}(k) \hat{\Theta}(k-1)) \\
\mathbf{P}(k)= & \frac{1}{\lambda}\left(\mathbf{P}(k-1)-\frac{\mathbf{P}(k-1) \mathbf{Z}(k) \mathbf{Z}^{T}(k) \mathbf{P}(k-1)}{\lambda+\mathbf{Z}^{T}(k) \mathbf{P}(k-1) \mathbf{Z}(k)}\right)
\end{aligned}
$$

where $Y$ is the output, $\boldsymbol{\Theta}$ is the unknown parameter vector of the model, $\mathbf{Z}$ is the input vector, $\mathbf{P}$ is the covariance matrix, and $\lambda$ is the forgetting factor. During the injection period, the $\mathrm{d}$-axis and q-axis inductances of the motor are estimated from the discrete model of the transient voltage equation as follows:

$$
\begin{aligned}
& Y_{L d}(k)=i_{d}(k)-i_{d}(k-1) \\
& Z_{L d}(k)=v_{d}(k)-R i_{d}(k-1)+L_{q} i_{q}(k-1) \omega(k-1) \\
& \quad \Theta_{L d}(k)=T_{s} / L_{d} \\
& Y_{L q}(k)=i_{q}(k)-i_{q}(k-1) \\
& Z_{L q}(k)=v_{q}(k)-R i_{q}(k-1)-\left\{L_{d} i_{d}(k-1)+\lambda_{p m}\right\} \omega(k-1)(11) \\
& \Theta_{L q}(k)=T_{s} / L_{q}
\end{aligned}
$$

where $T_{s}$ represents the sampling period. The output current 


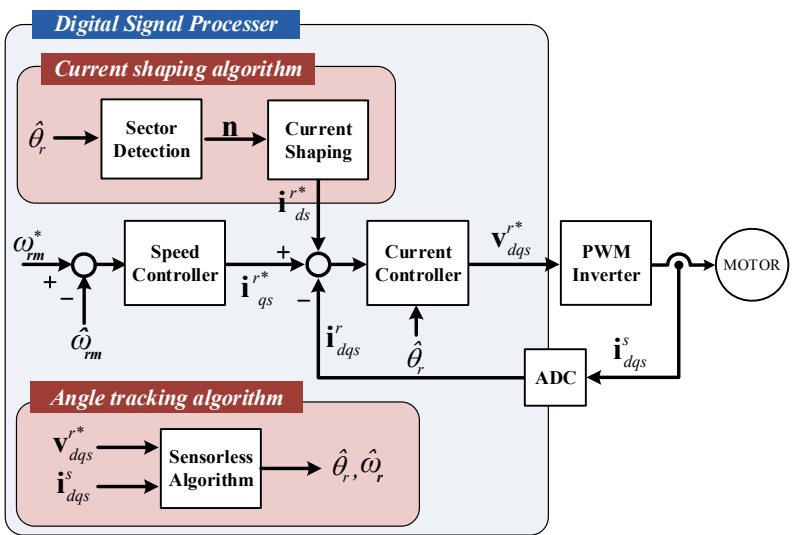

Fig. 9. Proposed control block diagram for the back EMF-based sensorless algorithm.

and voltage are assumed to be constant during the non-injection period. Hence, a derivative term of the transient voltage equation can be neglected, and the equation is simplified as follows:

$$
\begin{aligned}
& v_{d s}^{r}=\hat{R}_{s} i_{d s}^{r}-\omega_{r} \hat{L}_{q} i_{q s}^{r} \\
& v_{q s}^{r}=\hat{R}_{s} i_{q s}^{r}+\omega_{r}\left(\hat{L}_{d} i_{d s}^{r}+\hat{\lambda}_{p m}\right)
\end{aligned}
$$

From the above equations, the stator resistance and flux linkage are estimated with the discrete model as follows:

$$
\begin{aligned}
& Y_{R}(k)=v_{d}(k-1)+L_{q} I_{q}(k-1) \omega(k-1) \\
& Z_{R}(k)=i_{d}(k-1) \\
& \Theta_{R}(k)=R_{s} \\
& Y_{\text {lam }}(k)=v_{q}(k)-R i_{q}(k-1)-L_{d} I_{d}(k-1) \omega(k-1) \\
& Z_{\text {lam }}(k)=i_{q}(k-1) \\
& \Theta_{\text {lam }}(k)=\lambda_{\text {pm }}
\end{aligned}
$$

\section{Sensorless Algorithm}

The back EMF-based sensorless algorithm achieves a low performance in the low speed region because of the output voltage error. Hence, an additional high frequency signal injection is generally required for the sensorless drive in low speed regions. The proposed current shaping algorithm reduces output voltage errors and enhances the accuracy of angle estimation in low speed regions. The proposed control block diagram for the back EMF-based sensorless algorithm is similar to that for parameter estimation. As shown in Fig. 9, a block for current shaping is added to the conventional sensorless algorithm. In the low speed region, the proposed current shaping method is applied to reduce the output voltage errors. In the mid or high speed region, the proposed current injection is not required because of the sufficiently large output voltage.

\section{EXPERIMENTAL RESULTS}

The RLS-based parameter estimation method and back EMF-based sensorless algorithm are employed to verify the
TABLE II

PARAMETERS OF THE MOTOR AND TeSt CONDITIONS

\begin{tabular}{ccc}
\hline Meaning & Unit & Value \\
\hline Number of poles & - & 8 \\
Phase resistance & $\Omega$ & 9.16 \\
d-axis inductance & $\mathrm{mH}$ & 25.6 \\
q-axis inductance & $\mathrm{mH}$ & 25.6 \\
Flux linkage constant & $\mathrm{V} \cdot \mathrm{s} / \mathrm{rad}$ & 0.067 \\
\hline Dead time & $\mu \mathrm{s}$ & 1.5 \\
Switching frequency & $\mathrm{kHz}$ & 16 \\
DC link voltage & $\mathrm{V}$ & 310 \\
Test speed & $\mathrm{Rpm}$ & 75 \\
Load torque & $\mathrm{Nm}$ & 0.28 \\
\hline
\end{tabular}

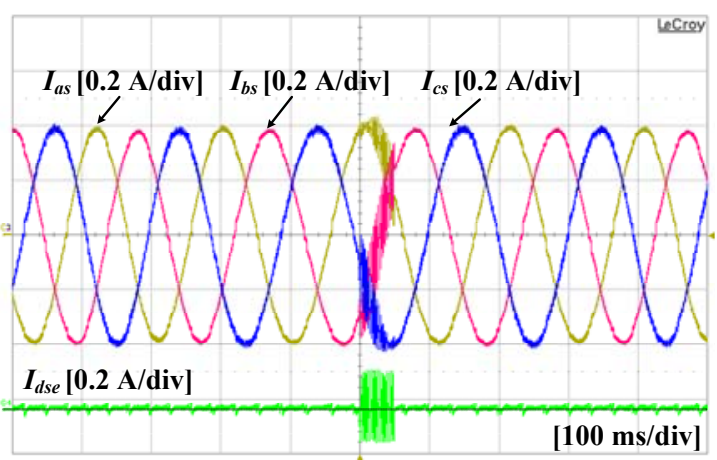

(a)

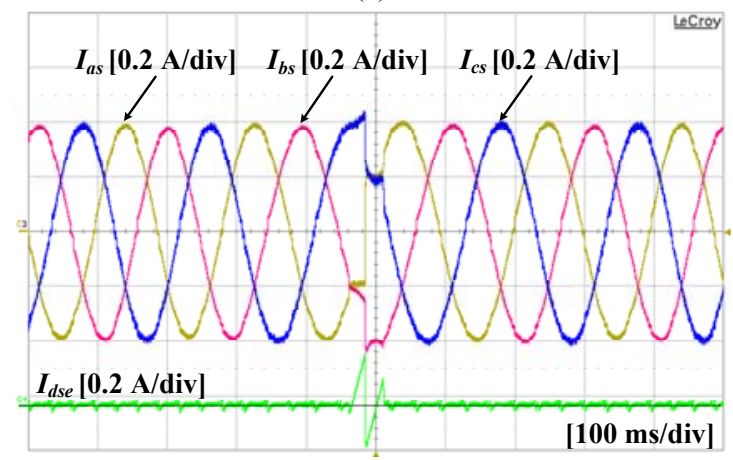

(b)

Fig. 10. Waveforms of each phase and d-axis current. (a) The conventional method. (b) The proposed method.

performance and feasibility of the proposed method, respectively. As shown in Table II, the stator resistance of the machine is $9.16 \Omega$, the inductance is $25.6 \mathrm{mH}$, and the flux linkage constant is $0.065 \mathrm{~V} \cdot \mathrm{s} / \mathrm{rad}$. The effect of the output voltage error in the low speed region is relatively more severe than that in the mid or high speed region. Hence, the experiment is performed at a low speed to show the enhancement of the parameters and angle estimation accuracy.

First, the experimental results demonstrate the performance of the parameter estimation method. The result of the proposed estimation method is compared with that of the conventional high frequency signal injection method. At $1 \mathrm{~s}$ intervals, each signal is injected for $50 \mathrm{~ms}$. Fig. 10(a) shows 


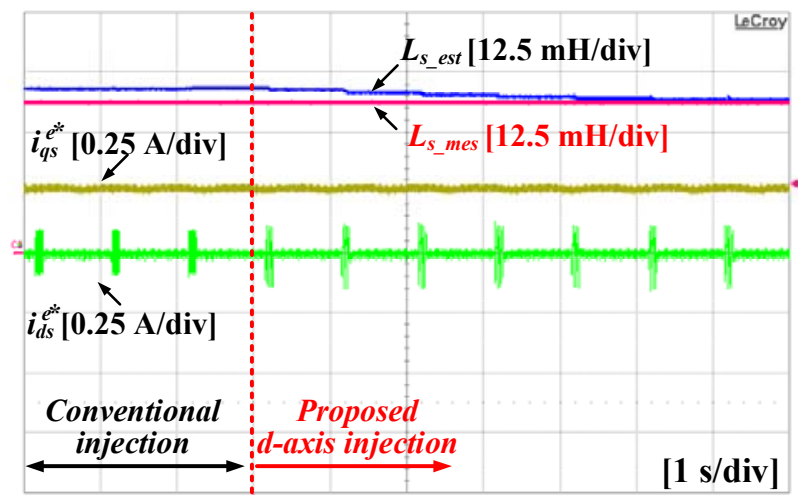

Fig. 11. Enhancement of estimation accuracy by the proposed d-axis injection.

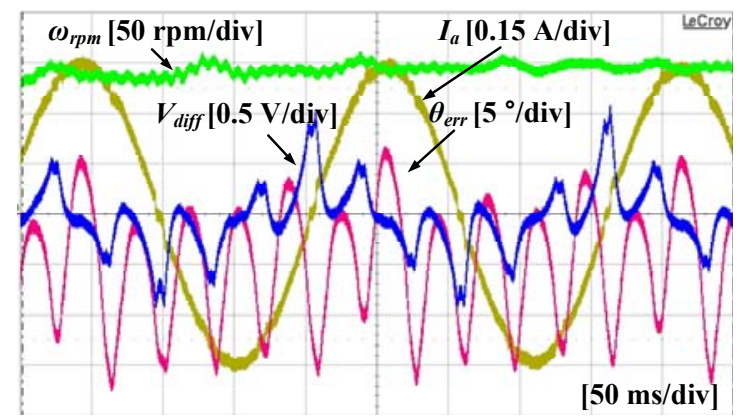

(a)

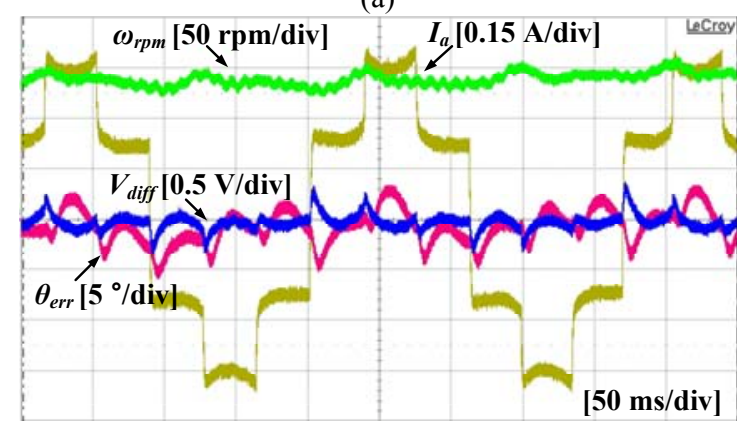

(b)

Fig. 12. Current waveform and voltage error. (a) Conventional method. (b) Proposed method.

the phase currents and d-axis current of the conventional method. The injection frequency is $300 \mathrm{~Hz}$, and the magnitude is $0.15 \mathrm{~A}$. The waveform of the phase current changes because of the injection. However, this change does not affect the estimation error reduction. Fig. 10(b) shows the phase currents and d-axis current of the proposed method. The injection signal is calculated from Equation (8) for the current shaping. The a-phase current increases dramatically during the injection, thereby reducing the estimation error. Fig. 11 shows the estimated inductance value, injected d-axis current reference, and q-axis current reference. First, the conventional injection method is applied. The proposed $\mathrm{d}$-axis injection is performed upon reaching the red dotted line. The estimated resistance and flux linkage constant represents similar values under both methods. However, the estimated inductance values are different. Fig. 11 shows that the inductance value estimated with the proposed method becomes similar to the measured inductance as a result of current shaping, whereas an error occurs with the conventional method because of the output voltage error. The convergence rate of the parameter estimation method is related to the forgetting factor of the RLS method, injection time, and proportional constant $k$ in (7). The convergence rate can increase with a long injection time and a large forgetting factor or proportional constant $k$. However, those values are adequately set in Fig. 11 to show the estimation procedure.

Second, the experimental results show the performance of the sensorless algorithm. The differences in the angle estimation capabilities of the conventional method and the proposed method are shown in Fig. 12. With the conventional method shown in Fig. 12(a), voltage and estimated angle errors occur at every zero crossing point. With the proposed method shown in Fig. 12(b), the errors in the output voltage and position estimation are reduced by more than $50 \%$.

\section{CONCLUSIONS}

This work proposes a method for reducing output voltage errors using the current shaping algorithm. In low speed regions, the output voltage errors caused by the nonlinearity of inverters are relatively severe. These errors can cause the accuracy of the parameter estimation method and sensorless algorithm to decrease. Thus, a new phase current waveform is proposed to reduce output voltage errors. This proposed current waveform can be easily implemented by adding a $\mathrm{d}$-axis current reference, which is similar to the sawtooth waveform. The proposed current shaping method injects this current waveform to improve accuracy. Furthermore, the change in the current reference does not affect the q-axis current reference, which is directly related to the output torque. Understandably, torque ripples can be generated in the interior permanent magnet synchronous motor (IPMSM) drive because of the additional d-axis current injection. However, the $\mathrm{d}$-axis injection is not always required. In parameter estimation, the $\mathrm{d}$-axis current is injected only for a short time, which is generally adjustable. Hence, the effect of the additional injection is negligible. In the case of the sensorless algorithm, the injection is continuously required. Therefore, the proposed method can be used only in low speed areas that do not serve as main operation areas. The RLS-based parameter estimation method and back EMF-based sensorless algorithm are implemented using an eight-pole SMPM machine in a low speed region to demonstrate the performance and feasibility of the proposed method.

\section{ACKNOWLEDGMENT}

This work was supported by the National Research 
Foundation of Korea (NRF) Grant funded by the Ministry of Science, ICT, \& Future Planning (MSIP) (2009-0083495) and the Brain Korea 21 Plus Project in 2015.

\section{REFERENCES}

[1] J.W. Kim, K. W. Kim, D. O. Kisck, D. H Kang, J. W Chang, and J. M. Kim, "A study on sensorless control of transverse flux rotating motor based on MRAS with parameter estimation," Journal of Power Electronics, Vol. 11, No. 6, pp. 864-869, Jun. 2011.

[2] K.W. Lee and J.-I. Ha, "Evaluation of back-EMF estimators for sensorless control of permanent magnet synchronous motors," Journal of Power Electronics, Vol. 12, No. 7, pp. 604-614, Jul. 2012.

[3] J.-I. Ha, K. Ide, T. Sawa, and S.-K. Sul, "Sensorless rotor position estimation of an interior permanent-magnet motor from initial states," IEEE Trans. Ind. Appl., Vol. 39, No. 3, pp. 761-767, May/Jun. 2003.

[4] G. Valverde, E. Kyriakides, G. T. Heydt, and V. Terzija, "Nonlinear estimation of synchronous machine parameters using operating data," IEEE Trans. Energy Convers., Vol. 26, No. 3, pp. 831-839, Sep. 2011.

[5] P. Niazi and H. A. Toliyat, "Online parameter estimation of permanent magnet assisted synchronous reluctance motor," IEEE Trans. Ind. Appl., Vol. 43, No. 2, pp. 609-615, Mar./Apr. 2007.

[6] A. Piippo, M. Hinkkanen, and J. Luomi, "Adaptation of motor parameters in sensorless PMSM drives," IEEE Trans. Ind. Appl., Vol. 45, No. 1, pp. 203-212, Jan./Feb. 2009.

[7] K. Liu, Q. Zhang, J. T. Chen, Z. Q. Zhu, J. Zhang, and A. W. Shen, "Online multi-parameter estimation of non-salient pole PM synchronous machines with temperature variation tracking," IEEE Trans. Ind. Electron., Vol. 58, No. 5, pp. 1776-1788, May 2011.

[8] Z. Q. Zhu, X. Zhu, and P. D. Sun, "Estimation of winding resistance and PM flux-linkage in brushless AC machines by reduced-order extended Kalman filter," in Proc. IEEE Int. Conf. Netw., Sens. Control, pp. 740-745, 2007.

[9] K. Liu, Z. Q. Zhu, Q. Zhang, and J. Zhang, "Influence of nonideal voltage measurement on parameter estimation in permanent magnet synchronous machines," IEEE Trans. Ind. Electron., Vol. 59, No. 6, pp. 2438-2447, Jun. 2012.

[10] L. M. Gong and Z. Q. Zhu "Modeling and compensation of inverter nonlinearity effects in carrier signal injection-based sensorless control methods from positive sequence carrier current distortion," in Proc. IEEE Energy Conversion. Congress and Exposition, pp. 3434-3441, 2010.

[11] J. W. Kim and J. I. Ha, "Enhancement of output voltage using current shaping in sensorless AC machine drive," Energy Conversion Congress and Exposition (ECCE), pp. 2490-2494, 2014.

[12] J. W. Kim and J. I. Ha, "Enhancement of parameter estimation accuracy using current shaping in PM machine drive," Power Electronics and ECCE Asia (ICPE-ECCE Asis), pp. 2123-2128, 2015.

[13] J.W. Choi and S.K. Sul, "A new compensation strategy reducing voltage current distortion in PWM VSI systems operating with low output voltages," IEEE Trans. Ind. Appl., Vol. 31, No. 5, pp. 1001-1008, Sep./Oct. 1995.
[14] S. Morimoto, M. Sanada, and Y. Takeda, "Mechanical sensorless drives of IPMSM with online parameter identification," IEEE Trans. Ind. Appl.,Vol. 42, No. 5, pp. 1241-1248, Sep./Oct. 2006.

[15] Y. Inoue, Y. Kawaguchi, S. Morimoto, and M. Sanada, "Performance improvement of sensorless IPMSM drives in a low-speed region using online parameter identification," IEEE Trans. Ind. Appl., Vol. 47, No. 2, pp. 798-804, Mar./Apr. 2011.

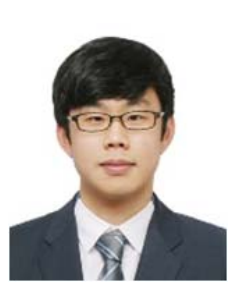

Jin-Woong Kim was born in Korea in 1986. In 2012, he received his bachelor's degree in electrical engineering from Seoul National University, Seoul, Korea, where he is currently working toward a doctor's degree. His current research interests include renewable energy conversion systems and battery management systems.

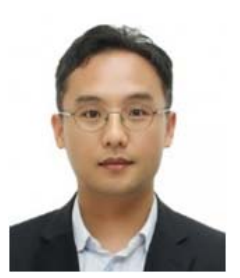

Jung-Ik Ha was born in Korea in 1971. He received his bachelor's, master's, and doctor's degrees in electrical engineering from Seoul National University, Seoul, Korea, in 1995, 1997, and 2001, respectively. $\mathrm{He}$ served as a researcher in Yaskawa Electric Co., Japan from 2001 to 2002. He was with Samsung Electronics Co., Korea as a senior and principal engineer from 2003 to 2008 . He was the chief technology officer of LS Mecapion Co., Korea from 2009 to 2010. Since 2010, he has been serving as an associate professor in the Department of Electrical and Computer Engineering, Seoul National University. His current research interests include high efficiency circuits and control and integrated electric energy conversions for various industrial fields. 